DOI: 10.15593/RZhBiomeh/2016.3.01

УДК 531/534:[57+61]
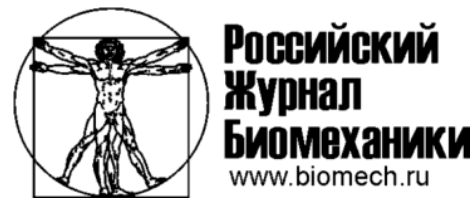

www.biomech.ru

\title{
О ПРИРОДЕ ВНУТРИБРЮШНОГО ДАВЛЕНИЯ
}

\author{
В.С. Туктамышев
}

Кафедра теоретической механики и биомеханики Пермского национального исследовательского политехнического университета, Россия, 614990, Пермь, Комсомольский проспект, 29, e-mail: helpinvader@list.ru

\begin{abstract}
Аннотация. Исследованию влияния внутрибрюшного давления на различные органы и системы человеческого организма посвящено множество научных работ. Вместе с тем до сих пор остается нераскрытым вопрос о природе внутрибрюшного давления. В научной литературе встречаются некоторые объяснения фризического смысла внутрибрюшного давления, однако единой концепции, позволяющей объяснить наличие давления в полости живота, не существует. В соответствии с этим целью представленной работы является анализ результатов различных исследований, направленных на изучение причин возникновения давления в брюшной полости. На основе экспериментальных данных, приведенных в литературных источниках, описывается существование градиента внутрибрюшного давления, обусловленного действием гравитации. Рассматривается простейшая модель стенки брюшной полости, позволяющая оценить взаимозависимость между внутрибрюшным давлением и напряжениями в брюшной стенке, вызванными сокращением eе мышц. Анализируется влияние изменения объема брюшного содержимого на давление в полости живота. Кроме того, исследуется вопрос о закономерностях распределения давления в брюшной полости. По результатам проведенного анализа формулируется вывод о том, что существование внутрибрюшного давления объясняется действием силы тяжести на брюшное содержимое, а также напряжениями в тканях брюшной стенки. При этом брюшную полость можно рассматривать как наполненный жидкостью контейнер с эластичной оболочкой, способной изменять свою фрорму.
\end{abstract}

Ключевые слова: внутрибрюшное давление, распределение давления, напряжения в тканях брюшной стенки.

\section{ВВЕДЕНИЕ}

Примерно полтора века назад E.J. Marrey (в 1863 г.), P. Burt (в 1870 г.) и E.C. Wendt (в 1876 г.) опубликовали первые исследования, посвященные изучению внутрибрюшного давления человека $[5,31]$. С тех пор было проведено множество клинических наблюдений и экспериментов, связанных с патологиями, возникающими при повышении внутрибрюшного давления (внутрибрюшной гипертензии). С помощью этих работ были выявлены причины возникновения внутрибрюшной гипертензии [21, $28]$ и ее патологическое влияние на сердечно-сосудистую $[9,19,30,32]$, дыхательную $[8,24]$ и мочевыделительную системы $[13,27]$.

Повышение давления в полости живота рассматривалось и с точки зрения биомеханики. Наибольший интерес в этом направлении представляет влияние внутрибрюшного давления на нагружение поясничного отдела позвоночного столба [3, $6,7,11,12,14,17,18]$ и напряженно-деформированное состояние тканей передней брюшной стенки $[10,15,16,25]$. 
Несмотря на большое количество проведенных исследований, не до конца ясным остается вопрос о природе внутрибрюшного давления. В мировой научной литературе можно встретить работы, посвященные объяснению данного вопроса, однако единого подхода к пониманию физического смысла внутрибрюшного давления на сегодняшний день не существует. Таким образом, целью представленной работы является анализ литературных данных, описывающих существование давления в полости живота с различных точек зрения, и формулировка единой концепции природы внутрибрюшного давления.

\section{ГРАВИТАЦИОННЫЙ ГРАДИЕНТ ВНУТРИБРЮШНОГО ДАВЛЕНИЯ}

Всемирным сообществом по изучению внутрибрюшной гипертензии [33] внутрибрюшное давление определяется как установившееся давление, заключенное в брюшной полости. Такое утверждение считается достаточным среди специалистов, занимающихся патологиями полости живота. Вместе с тем, очевидно, что приведенное определение не раскрывает физический смысл внутрибрюшного давления. Частичное решение этой проблемы можно найти в работах физиологов, первая из которых была опубликована в 1946 г. [26]. В данной статье автором был проведен ряд экспериментов по измерению внутрибрюшного давления у собак, поддерживаемых в вертикальном положении. Измерения осуществлялись с помощью системы, включающей в себя накачиваемый воздухом резиновый баллон, соединенный с манометром посредством гибкой трубки. В ходе проведения опытов баллон через хирургические надрезы помещался в брюшную полость животного и устанавливался на различных по высоте уровнях (рис. 1). При этом давление, регистрируемое манометром (т.е. давление, оказываемое на баллон со стороны содержимого брюшной полости), принималось за внутрибрюшное давление. Регистрация внутрибрюшного давления $P$ в каждом случае сопровождалась измерением расстояния $h$ от верхней точки купола диафрагмы до баллона. Следует отметить, что в качестве описания параметра $h$ в излагаемой работе был предложен термин «высота столба брюшного содержимого» (the height of the column of the intra-abdominal contents [26]). Сопоставление измеренных величин $h$ и $P$ было представлено в виде соответствующего графика (рис. 2, a). Регрессионная линия, изображенная на данном графике, отражает зависимость между внутрибрюшным давлением и высотой столба брюшного содержимого над той точкой полости живота, в которой это давление было измерено (коэффициент корреляции $r=0,834$ ).

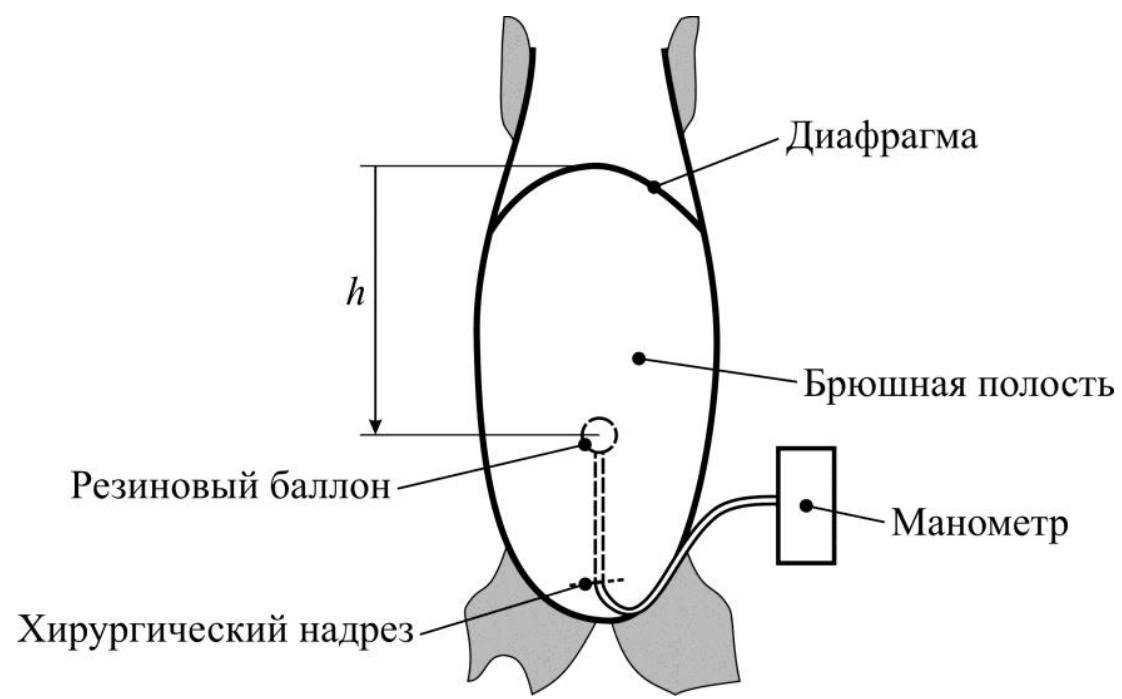

Рис. 1. Схема брюшной полости собаки с введенным баллоном [26] 

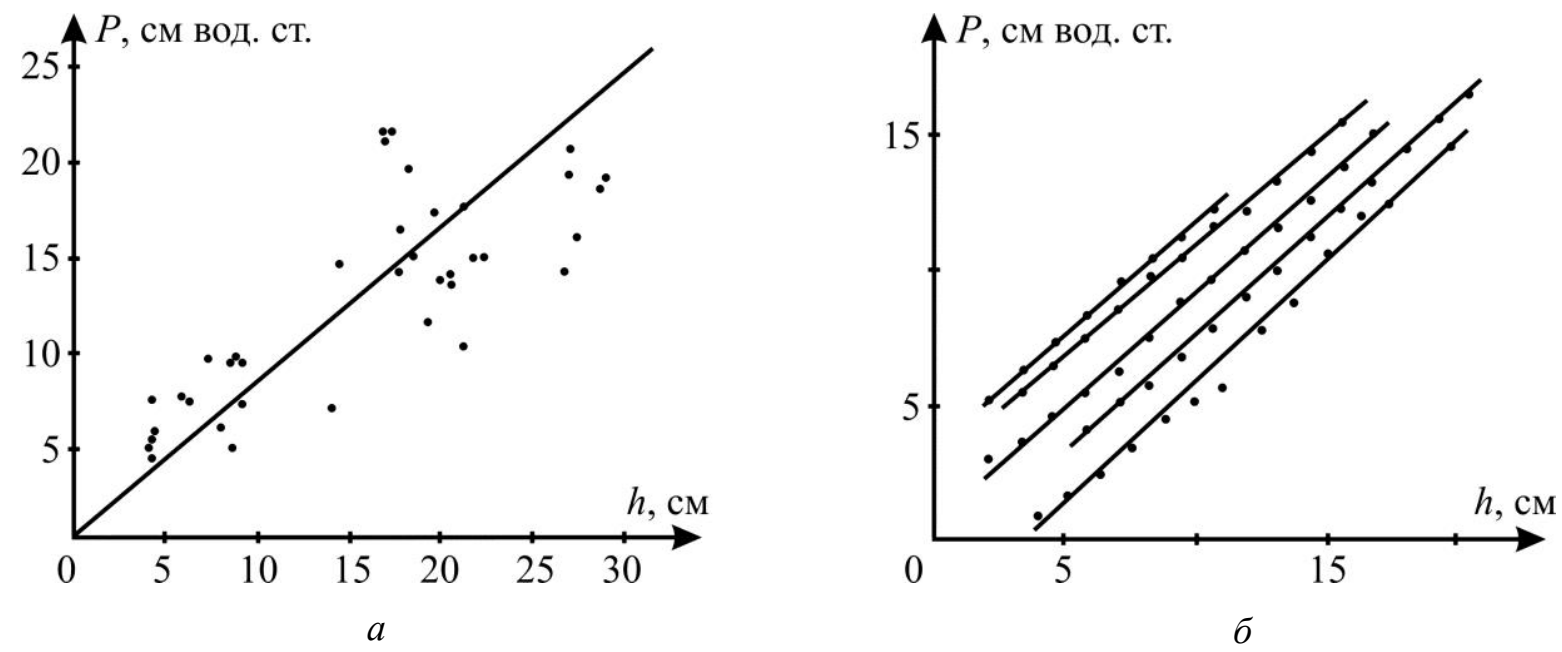

Рис. 2. Результаты исследований: $a-[26] ; \sigma-[20] . ~ P$ - внутрибрюшное давление, $h$ - расстояние от мечевидного отростка грудины до точки, в которой производится измерение

Исходя из полученных экспериментальных данных автор описываемой работы сформулировал вывод о том, что наличие давления в произвольной точке брюшного пространства обусловлено весом вышележащих подвижных органов полости живота. При этом величина регистрируемого внутрибрюшного давления напрямую соотносится с высотой столба брюшного содержимого над той точкой, в которой производится измерение.

Из графика, приведенного на рис. 2, a, видно, что некоторые экспериментальные точки находятся на достаточном отдалении от регрессионной линии. Это привлекло внимание скептиков, усомнившихся в справедливости ранее упомянутых выводов. В 1994 г. была опубликована работа [20], основанная на предположении о том, что дискуссии о результатах исследования [26] бессмысленны, поскольку для манометрической системы, которая была использована, характерна большая погрешность измерения. Данная погрешность связана с тем, что наполненный воздухом резиновый баллон имеет достаточно большой объем (около $10 \mathrm{~cm}^{3}$ ), способствующий возникновению локальных изменений в распределении давления в полости живота [20]. В этой же работе авторы провели экспериментальное исследование по измерению внутрибрюшного давления у собак, находящихся в состоянии апноэ. Все измерения были выполнены согласно схеме, принятой в статье [26]. Для повышения точности результатов экспериментов использовалась водно-перфузионная манометрическая система, широко применяемая в различных областях современной медицины. Вместо резинового баллона данная система включает в себя гибкую перфорированную трубку, диаметр которой не превышает 2 мм. Результаты исследования представлены в виде графика на рис. 2, б.

Следует отметить, что каждая регрессионная линия, показанная на графике, соответствует измерениям, выполненным на отдельном животном. Согласно экспериментальным данным, приведенным на рис. 2, б, внутрибрюшное давление практически линейно возрастает с увеличением высоты $h$. Таким образом, результаты излагаемого исследования подтвердили выводы, сформулированные в работе [26].

В дополнении к вышеописанным публикациям необходимо упомянуть о работе [29], авторы которой обратили внимание на вопрос об однородности распределения давления в брюшной полости человека. Для изучения этого вопроса в указанном исследовании была проведена серия экспериментов по измерению внутрибрюшного давления в четырех сегментах толстого кишечника: прямой, сигмовидной, нисходящей ободочной и поперечно-ободочной кишках (рис. 3). 


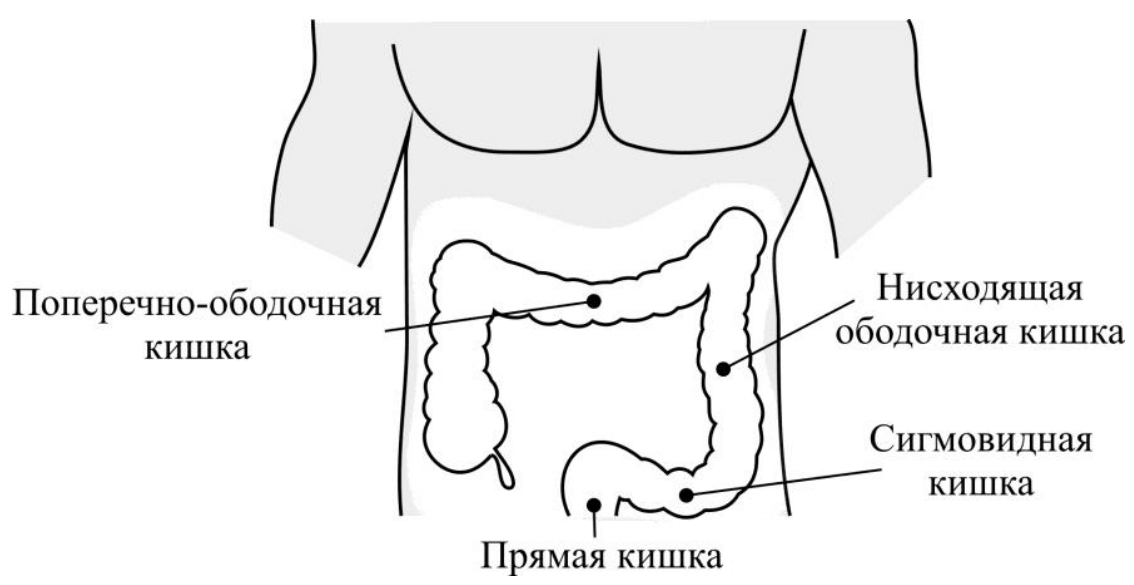

Рис. 3. Сегменты толстого кишечника, в которых согласно работе [29] проводились измерения внутрибрюшного давления

При выполнении измерений пациенты находились в положении лежа на спине. В таком положении регистрация внутрибрюшного давления осуществлялась в точках, находящихся приблизительно на одном (по высоте) уровне, т.е. из рассмотрения был исключен градиент внутрибрюшного давления, возникающий под действием гравитации. Кроме того, для исключения влияния давления перистальтической волны, распространяющейся вдоль стенок толстого кишечника, все измерения производились в периоды отсутствия перистальтики. По результатам экспериментов с участием пяти пациентов были вычислены средние значения давления в исследуемых отделах толстого кишечника. Полученные данные были оформлены в виде диаграммы (рис. 4), отражающей отношения величин $P_{\text {п }}, P_{\mathrm{H}}, P_{\mathrm{c}}$ и

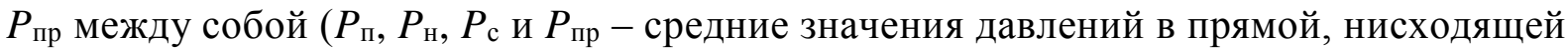
ободочной, сигмовидной и поперечно-ободочной кишках соответственно).

Согласно приведенным результатам отношения между найденными величинами давлений находятся в интервале от 1 до 1,1. Это значит, что распределение давления на определенном по высоте уровне брюшной полости можно считать однородным. Данное заключение в сочетании с результатами работ [20] и [26] привело авторов исследования [29] к выводу о том, что брюшная полость человека аналогична контейнеру, наполненному жидкостью, которая подчиняется законам гидростатики, причем давление этой жидкости (иными словами, внутрибрюшное давление) обусловлено действием гравитации.

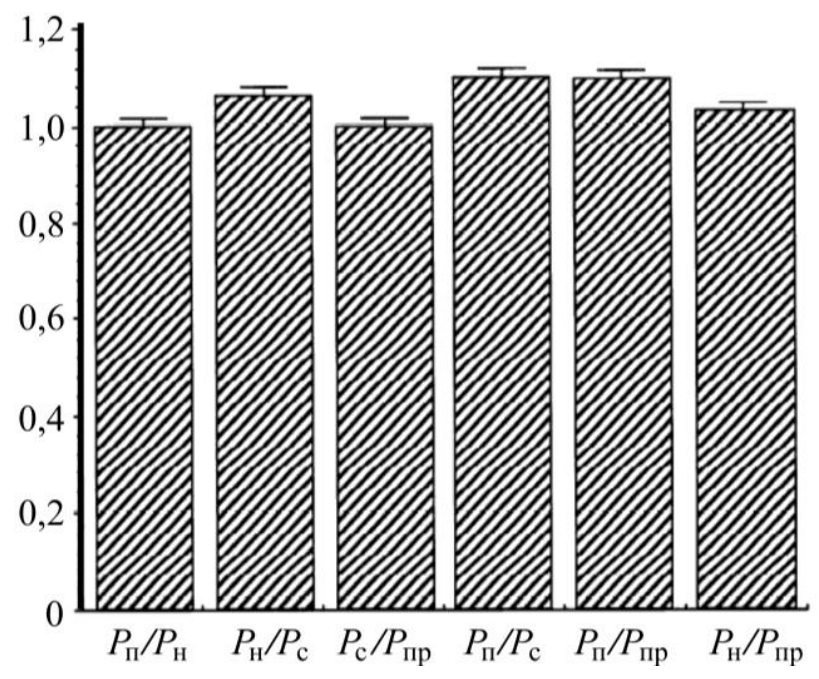

Рис. 4. Результаты исследования [29] 


\section{ВНУТРИБРЮШНОЕ ДАВЛЕНИЕ, ВОЗНИКАЮЩЕЕ ПРИ СОКРАЩЕНИИ МЫШЦ БРЮШНОЙ СТЕНКИ}

Несмотря на экспериментальное доказательство «гравитационной природы» внутрибрюшного давления, вес брюшного содержимого является не единственной причиной наличия давления в полости живота. Об этом свидетельствует, например, работа [22], в которой было показано, что внутрибрюшное давление при смехе может достичь значения в 70 мм рт. ст., при кашле - 120 мм рт. ст., при подъеме штанги (у тяжелоатлетов) - 375 мм рт. ст. Принимая во внимание стандартные величины внутрибрюшного давления для здорового взрослого человека (не превышающие в норме 7 мм рт. ст. [33]), можно утверждать, что указанные значения не могут быть обусловлены только влиянием гравитации. Разнообразные движения, выполняемые человеком в процессе жизнедеятельности, сопровождаются напряжением мышц брюшной стенки (в частности прямой, поперечной и косых мышц живота [2]). При сокращении волокон этих мышц оболочка полости живота стремится к уменьшению своего объема, что приводит к дополнительному давлению со стороны стенок полости на брюшное содержимое. Таким образом, высокие значения внутрибрюшного давления, возникающие, например, при кашле или подъеме тяжестей, объясняются значительными усилиями в мышцах брюшной стенки.

Для исследования взаимосвязи между внутрибрюшным давлением и напряжениями, возникающими в брюшной стенке за счет сокращения ее мышц, в работе [12] была предложена простейшая модель брюшной полости. Согласно данной модели брюшная стенка представляет собой тонкостенную осесимметричную упругую оболочку (рис. 5, a), для которой характерно уравнение Лапласа [1]

$$
\frac{\sigma_{m}}{\rho_{m}}+\frac{\sigma_{\theta}}{\rho_{\theta}}=\frac{p}{\delta},
$$

где $\rho_{m}-$ меридиональный радиус кривизны оболочки в некоторой точке (меридиональная плоскость, в которой вводится радиус $\rho_{m}$, изображена на рис. $5, a$ ); $\rho_{\theta}-$ радиус кривизны в плоскости, перпендикулярной меридиану; $\sigma_{m}$ и $\sigma_{\theta}-$ меридиональное и окружное напряжения в данной точке; $p$ - давление внутри оболочки; $\delta$ - толщина оболочки. Разумеется, давление $p$ в рамках предлагаемой модели соответствует внутрибрюшному давлению. Следует отметить, что в исследовании [12] не учитывался градиент внутрибрюшного давления, возникающий за счет действия гравитации.
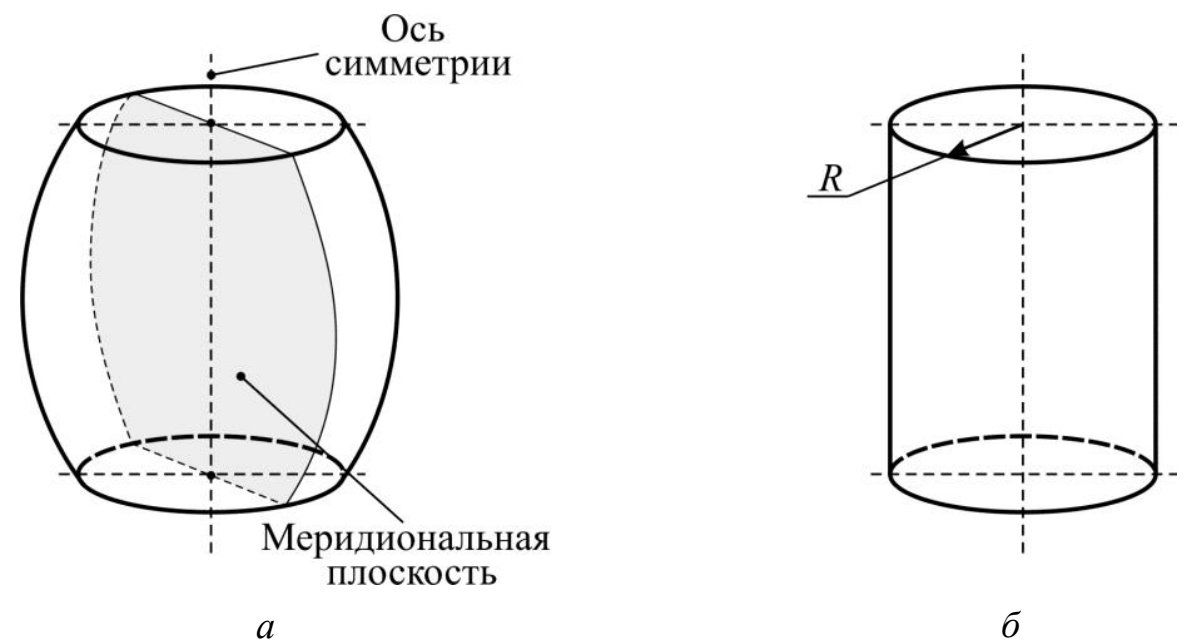

Рис. 5. Простейшая модель брюшной полости: $a$ - осесимметричная оболочка произвольной формы; $\sigma$ - оболочка цилиндрической формы $(R-$ радиус цилиндра) 
Авторами работы [12] был рассмотрен случай, когда $\rho_{m} \rightarrow \infty$. Очевидно, что форма оболочки при этом является цилиндрической (рис. 5, б). Согласно равенству (1) в данном случае значение внутрибрюшного давления определяется окружными напряжениями, реализуемыми в брюшной стенке:

$$
p=\sigma_{\theta} \delta / R \text {. }
$$

\section{ЗАВИСИМОСТЬ ВНУТРИБРЮШНОГО ДАВЛЕНИЯ ОТ ОБЪЕМА БРЮШНОГО СОДЕРЖИМОГО}

При увеличении объема брюшного содержимого передняя и боковая части брюшной стенки растягиваются, что можно наблюдать, например, при беременности. Способность к указанному растяжению зависит от множества факторов, и для каждого человека она является индивидуальной. Чрезмерное растяжение может сопровождаться нарушением целостности тканей стенки, проявляемым в виде грыжи живота. В этом случае нагрузки, действующие в области разрушения, превышают критические значения. Таким образом, изменение объема содержимого находится во взаимной зависимости с напряженным состоянием тканей брюшной стенки и, следовательно, с внутрибрюшным давлением.

Экспериментальное изучение описываемой зависимости проведено в работе [23]. В данном исследовании изменение брюшного объема достигалось посредством накачивания углекислого газа в брюшную полость испытуемого. При этом внутрибрюшное давление измерялось с помощью стандартного метода (через мочевой пузырь [4, 33]). Согласно результатам измерений искомая зависимость близка к линейной (рис. 6), причем авторы исследования подчеркивают, что подобная линейная тенденция наблюдалась у каждого испытуемого (всего в экспериментах приняли участие 120 добровольцев) [23].

Из приведенных рассуждений следует, что связь между внутрибрюшным давлением $P$ и объемом брюшного содержимого $V$ можно представить функцией

$$
P=k V+P_{0},
$$

где $k$ - коэффициент пропорциональности; $P_{0}$ - начальное давление.

Несмотря на простой вид функции (2), ее построение связано с большими трудностями, так как определение коэффициента $k$ в случае конкретного пациента является сложной экспериментальной проблемой. Вместе с тем установление зависимости (2) представляет собой важнейшую задачу в профилактике внутрибрюшной гипертензии, поскольку данная закономерность позволяет вычислить внутрибрюшное давление при увеличении объема брюшного содержимого. Этим объясняется актуальность разработки альтернативных алгоритмов, направленных на определение коэффициента $k$.

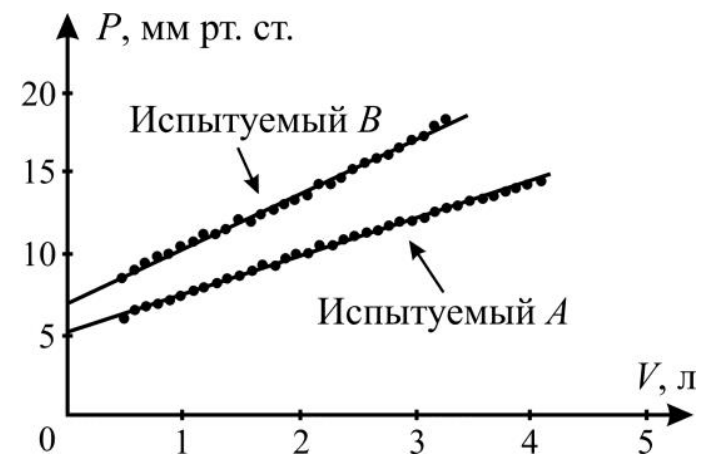

Рис. 6. Зависимость внутрибрюшного давления $P$ от объема $V$ брюшного содержимого для двух испытуемых [23]. Точками обозначены результаты измерений, отрезками - соответствующие линии регрессии 
Исходя из вышесказанного можно сделать следующий общий вывод. Наличие давления в брюшном пространстве обусловлено несколькими причинами: с одной стороны, силой тяжести, действующей на содержимое полости живота, с другой сокращением мышц брюшной стенки, с третьей - напряжениями в тканях брюшной оболочки, возникающими в результате увеличения ее объема. При этом брюшную полость можно рассматривать как наполненный жидкостью контейнер, оболочка которого является эластичной и способна изменять свою форму за счет активных сил, аналогичных мышечным усилиям.

\section{Выводы}

1. Существование давления в полости живота объясняется несколькими факторами, а именно: 1) действием силы тяжести на содержимое брюшной полости; 2) сокращением мышц брюшной стенки; 3) наличием напряжений в тканях брюшной стенки при увеличении объема полости.

2. Распределение внутрибрюшного давления на некотором уровне (по высоте) брюшной полости можно считать однородным.

3. В качестве модели брюшной полости можно рассматривать контейнер, наполненный жидкостью. При этом эластичная оболочка контейнера способна изменять свою форму за счет действия активных сил, аналогичных усилиям мышц брюшной стенки.

\section{БЛАГОДАРНОСТИ}

Автор благодарит профессора С. МакГилла (Университет Ватерлоо, г. Ватерлоо, Канада) и К. Даггфельдта (Шведская школа спорта и медицинских наук, г. Стокгольм, Швеция) за полезные обсуждения в ходе работы над статьей.

\section{СПИСОК ЛИТЕРАТУРЫ}

1. Дарков А.В., Шпиро Г.С. Сопротивление материалов. - М.: Высшая школа, 1975. - 654 с.

2. Синельников Р.Д. Атлас анатомии человека: в 3 т. - М.: Медгиз, 1963. - Т. 1. - 477 с.

3. Туктамышев В.С., Безматерных В.В. Моделирование влияния внутрибрюшного давления на нагружение позвоночного столба // Современные проблемы науки и образования. - 2014. - № 3. C. 704 .

4. Туктамышев В.С., Касатова Е.Ю., Няшин Ю.И. Исследование зависимости между давлением выдыхаемого воздуха и внутрибрюшным давлением человека // Российский журнал биомеханики. 2015. - Т. 19, № 1. - С. 73-78.

5. Туктамышев В.С., Кучумов А.Г., Няшин Ю.И., Самарцев В.А., Касатова Е.Ю. Внутрибрюшное давление человека // Российский журнал биомеханики. - 2013. - Т. 17, № 1. - С. 22-31.

6. Туктамышев В.С., Соломатина Н.В. Влияние внутрибрюшного давления на состояние поясничного отдела позвоночника // Фундаментальные исследования. - 2013. - № 8. - С. 77-81.

7. Bartelink D.L. The role of abdominal pressure in relieving the pressure on the lumbar intervertebral discs // Journal of Bone and Joint Surgery. - 1957. - Vol. 39. - P. 718-725.

8. Bloomfield G.L., Ridings P.C., Blocher C.R. A proposed relationship between increased intra-abdominal, intrathoracic and intracranial pressure // Critical Care Medicine. - 1997. - Vol. 25, № 3. - P. 496-503.

9. Caldwell C.B., Ricotta J.J. Changes in visceral blood flow with elevated intra-abdominal pressure // Journal of Surgery Research. - 1987. - Vol. 43. - P. 14-20.

10. Calvo B., Sierra M., Grasa J., Munoz M.J., Pena E. Determination of passive viscoelastic response of the abdominal muscle and related constitutive modeling: Stress-relaxation behavior // Journal of Mechanical Behavior of Biomedical Materials. - 2014. - Vol. 36. - P. 47-58.

11. Cholewicki J., Juluru K., McGill S.M. Intra-abdominal pressure mechanism for stabilizing the lumbar spine // Journal of Biomechanics - 1999. - Vol. 32. - P. 13-17.

12. Daggfeldt K., Thorstensson A. The role of intra-abdominal pressure in spinal unloading // Journal of Biomechanics. - 1997. - Vol. 30, № 11/12. - P. 1149-1155.

13. Doty J.M., Saggi B.H., Blocher C.R., Fakhry I., Gehr T., Sica D., Sugerman H.J. Effects of increased renal 
parenchymal pressure on renal function // Journal of Trauma. - 2000. - Vol. 48, № 5. - P. 874-877.

14. Essendrop M., Andersen T.B., Schibye B. Increase in spinal stability obtained at levels of intra-abdominal pressure and back muscle activity realistic to work situations // Applied Ergonomics. - 2002. - Vol. 33. P. 471-476.

15. Hernandez B., Pena E., Pascual G., Rodriguez M., Calvo B., Doblare M., Bellon J.M. Mechanical and histological characterization of the abdominal muscle. A previous step to model hernia surgery // Journal of Mechanical Behavior of Biomedical Materials. - 2011. - Vol. 4. - P. 392-404.

16. Hernandez-Gascon B., Mena A., Pena E., Pascual G., Bellon J.M., Calvo B. Understanding the passive mechanical behavior of the human abdominal wall // Annals of Biomedical Engineering. - 2013. - Vol. 41, № 2. - P. 433-444.

17. Hodges P.W., Cresswell A.G., Daggfeldt K., Thorstensson A. In vivo measurement of the effect of intraabdominal pressure on the lumbar spine // Journal of Biomechanics. - 2001. - Vol. 34. - P. 347-353.

18. Hodges P.W., Eriksson A.E., Shirley D., Gandevia S.C. Intra-abdominal pressure and abdominal wall muscular function: spinal unloading mechanism // Journal of Biomechanics. - 2005. - Vol. 38. - P. 18731880 .

19. Kuntscher M.V., Germann G., Hartmann B. Correlations between cardiac output, stroke volume, central venous pressure, intra-abdominal pressure and total circulating blood volume in resuscitation of major burns // Resuscitation. - 2006. - Vol. 70, № 1. - P. 37-43.

20. Loring S.H., Yoshino K., Kimball W.R., Barnas G.M. Gravitational and shear-associated pressure gradients // Journal of Applied Physiology. - 1994. - Vol. 77, № 3. - P. 1375-1382.

21. Malbrain M.L., Chiumello D., Pelosi P., Bihari D., Innes R., Ranieri V.M., Del Turco M., Wilmer A., Brienza N., Malcangi V., Cohen J., Japiassu A., De Keulenaer B.L., Daelemans R., Jacquet L., Laterre P.F., Frank G., De Souza P., Cesana B., Gattinoni L. Incidence and prognosis of intra-abdominal hypertension in a mixed population of critically ill patients: A multiple-center epidemiological study // Critical Care Medicine. - 2005. - Vol. 33, № 2. - P. 315-322.

22. Mens J., Van Dijke G.H., Pool-Goudzwaard A., Van Der Hulst V., Stam H. Possible harmful effects of high intra-abdominal pressure on the pelvic girdle // Journal of Biomechanics. - 2006. - Vol. 39, № 4. P. 627-635.

23. Mulier J.P., Dillemans M., Crombach C., Missant C., Sels A. On the abdominal pressure volume relationship [Электронный ресурс] // The Internet Journal of Anesthesiology. - 2009. - Vol. 21, № 1. URL: www.ispub.com/IJA/21/1/5221) (дата обращения: 21.07.2016).

24. Obeid F., Saba A., Fath J. Increases in intra-abdominal pressure affect pulmonary compliance // Archives of Surgery. - 1995. - Vol. 130. - P. 544-548.

25. Pachera P., Pavan P.G., Todros S., Cavinato C., Fontanella C.G., Natali A.N. A numerical investigation of the healthy abdominal wall structures // Journal of Biomechanics. - 2016. - Vol. 49, № 9. - P. 1818-1823.

26. Rushmer R.F. The nature of intraperitoneal and intrarectal pressures // American Journal of Physiology. 1946. - Vol. 146. - P. 242-249.

27. Shenansky J.H., Gillenwater J.Y. The renal hemodynamic and functional effects of external counterpressure // Surgery, Gynecology \& Obstetrics. - 1972. - Vol. 134. - P. 253-258.

28. Surgue M. Abdominal compartment syndrome // Current Opinion in Critical Care. - 2005. - Vol. 11, N. 4. - P. 333-338.

29. Tzelepis G.E., Nasiff L., McColl F.D., Hammond J. Transmission of pressure within the abdomen // Journal of Applied Physiology. - 1996. - Vol. 81, № 3. - P. 1111-1114.

30. Valenza F., Irace M., Guglielmi M., Gatti S., Bottino N., Tedesco C., Maffioletti M., Maccagni P., Fossali T., Aletti G., Gattinoni L. Effects of continuous negative extra-abdominal pressure on cardiorespiratory function during abdominal hypertension: an experimental study // Intensive Care Medicine. - 2005. - Vol. 31, № 1. - P. 105-111.

31. Van Hee R. Historical highlights in concept and treatment of abdominal compartment syndrome // Acta Clinica Belgica. - 2007. - Vol. 62. - S9-S15.

32. Vivier E., Metton O., Piriou V., Lhuillier F., Cottet-Emard J.M., Branche P., Duperret S., Viale J.P. Effects of increased intra-abdominal pressure on central circulation // British Journal of Anaesthesia. - 2006. Vol. 96, № 6. - P. 701-707.

33. World Society of the Abdominal Compartment Syndrome [Электронный pecypc]. - URL: www.wsacs.org (дата обращения: 30.07.2016). 


\section{ON THE NATURE OF INTRA-ABDOMINAL PRESSURE}

\section{V.S. Tuktamyshev (Perm, Russia)}

A lot of scientific papers is dedicated to study of the influence of intra-abdominal pressure on the different organs and systems of a human body. At the same time, a question on the nature of intra-abdominal pressure still remains unsolved. In the scientific literature, there are some explanations of the physical meaning of intra-abdominal pressure, but the complete concept, which allows us to explain the presence of pressure in the abdomen, does not exist. In accordance with this, the aim of the present work is to analyze the results of the different studies aimed at studying causes of emergence of the pressure in the abdominal cavity. On the basis of the experimental data, presented in literary sources, this article describes the existence of gradient of intra-abdominal pressure, caused by the effect of gravity. We examine the simplest model of the abdominal wall in order to estimate the relationship between intra-abdominal pressure and stresses in the wall caused by the contraction of muscles of the abdominal wall. We also analyze the influence of changes of the volume of abdominal contents on the pressure in the abdominal cavity. In addition, we investigate the question of trends of the distribution of pressure in the abdomen. As a result of the conducted analysis, we formulate a conclusion that the existence of intra-abdominal pressure is explained by the effect of gravity on the abdominal contents and the stresses in the abdominal wall tissues. In this way, the abdominal cavity can be regarded as a liquid-filled container with an elastic sheath that can change its shape.

Key words: intra-abdominal pressure, pressure distribution, stresses in abdominal wall tissues. 